\title{
Hypocalcaemia Following Laryngectomy
}

Research Question: what is the prevalence of hypocalcaemia after laryngectomy and is total thyroidectomy a risk factor? \section{Andrew S Harris Eduard Prades Carl D Passant
Aneurin Bevin University Health Board, The Royal Gwen $\mathbb{Z}_{\text {Hospital, Newport }}$}

\begin{tabular}{|c|c|c|c|c|c|c|}
\hline \multirow{2}{*}{ 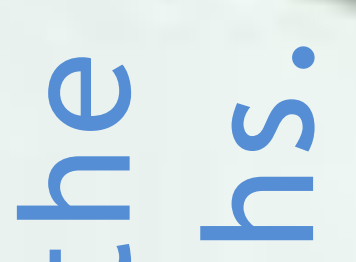 } & \multirow{3}{*}{$\begin{array}{l}\text { Hypocalcaemia is a recognised } \\
\text { complication of laryngectomy. } \\
\text { However, there is a scarcity of }\end{array}$} & \multicolumn{5}{|c|}{ Patient Demographics } \\
\hline & & & $\begin{array}{l}\text { Thyroid in } \\
\text { situ }\end{array}$ & $\begin{array}{c}\text { Hemi } \\
\text { thyroidectomy }\end{array}$ & $\begin{array}{c}\text { Total } \\
\text { thyroidectomy }\end{array}$ & Total \\
\hline 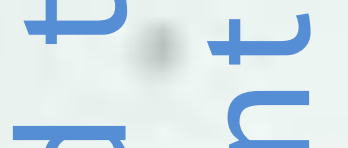 & & Total & 14 & 69 & 7 & 90 \\
\hline$\overline{0}$ & research in this area. The & Male & & & & $83 \%$ \\
\hline $\bar{\sigma} \varepsilon$ & $\begin{array}{l}\text { prevalence and risk factors are } \\
\text { unknown. }\end{array}$ & Neck dissection & $71 \%$ & $99 \%$ & $100 \%$ & $92 \%$ \\
\hline$\frac{\varepsilon}{\sigma}$ & Postoperative hypocalcaemia & $\begin{array}{c}\text { Salvage } \\
\text { Procedure }\end{array}$ & $71 \%$ & $29 \%$ & $14 \%$ & $34 \%$ \\
\hline$\frac{2}{\varepsilon}$ & $\begin{array}{l}\text { causes extended admission } \\
\text { (often to intensive care), }\end{array}$ & $\begin{array}{c}\text { Adjuvant } \\
\text { Radiotherapy }\end{array}$ & $29 \%$ & $54 \%$ & $86 \%$ & $52 \%$ \\
\hline
\end{tabular}
requires long lines, blocks NG tubes, needs repeated blood tests and results in lifelong reliance on oral medication.

\section{Post laryngectomy}

hypocalcaemia results in extra morbidity and increased health costs.

Consecutive total laryngectomies at The Royal Gwent Hospital identified from January 2006 to August 2017.
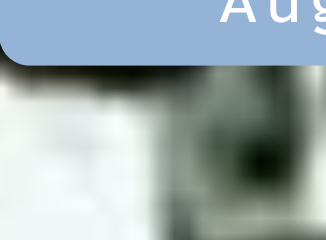

Electronic records retrospectively reviewed for operation notes, letters and investigation results. (W)

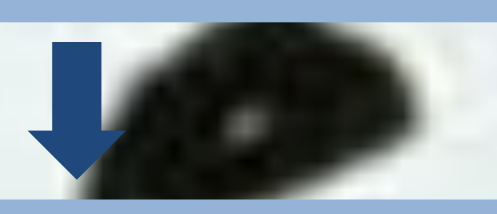

Analysis of results for early (12-24 hours), protracted (4-6 weeks) and permanent hypocalcaemia (after 1 year).
Prevalence of hypocalcaemia

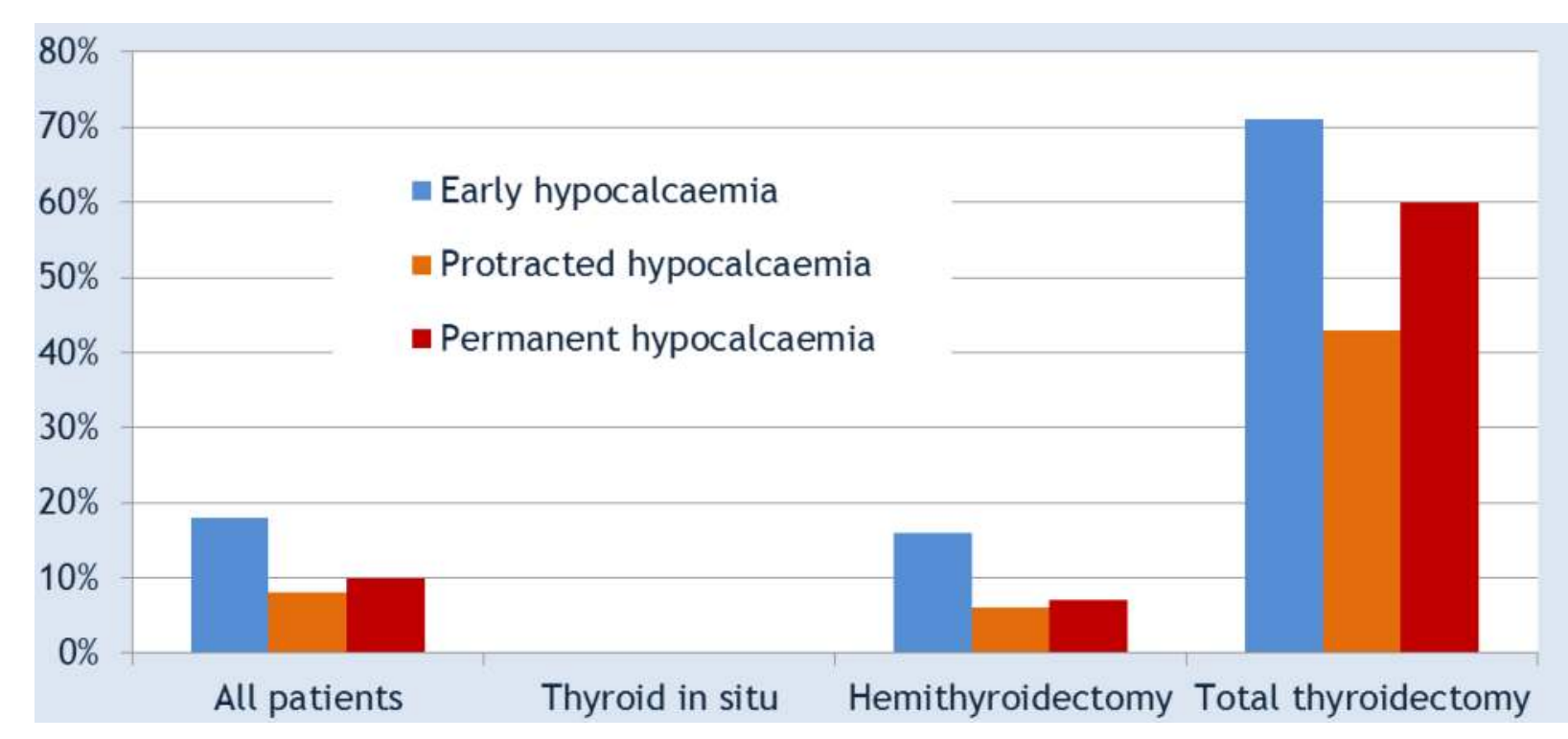

Exact logistic regression analysis shows that total thyroidectomy was significant at all time frames. Other variables did not have a significant effect.

\begin{tabular}{ccccc}
\hline & $\begin{array}{c}\text { Number } \\
\text { of patients }\end{array}$ & Odds ratio & $95 \% \mathrm{Cl}$ & $\begin{array}{c}\text { Model P- } \\
\text { value }\end{array}$ \\
\hline $\begin{array}{c}\text { Early } \\
\text { hypocalcaemia } \\
\begin{array}{c}\text { Protracted } \\
\text { hypocalcaemia }\end{array}\end{array}$ & 90 & 15.5 & $2.2-181.9$ & 0.002 \\
$\begin{array}{c}\text { Permanent } \\
\text { hypocalcaemia }\end{array}$ & 60 & 13.3 & $1.5-117.1$ & 0.010 \\
\hline
\end{tabular}

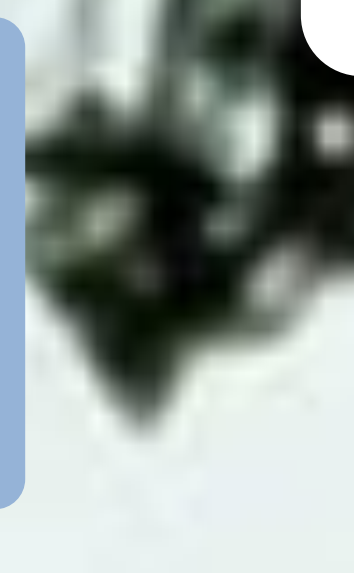

hypocalcaemia

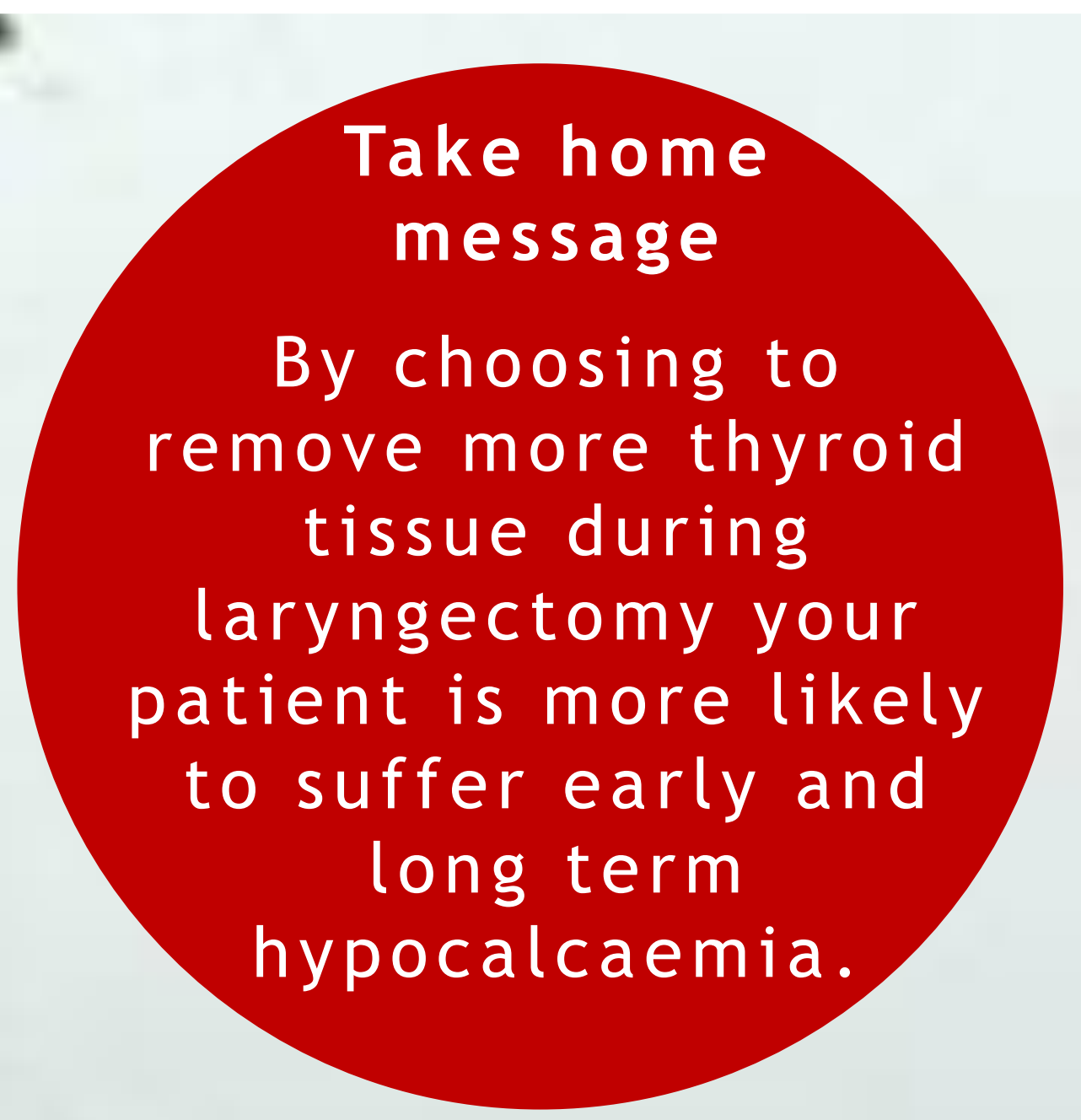

